\title{
Ray-Scaling Operations for Deformation of Soft Objects
}

\author{
Pi-Chung Hsu* \\ Department of Information Management, Shu-Te University, Kaohsiung City, Taiwan. \\ * Corresponding author. Tel: 886-76158000; email: pichung@stu.edu.tw \\ Manuscript submitted September 24, 2015; accepted December 10, 2015. \\ doi: $10.17706 /$ jcp.11.6.520-527
}

\begin{abstract}
In soft object modeling, in order to make the shapes of primitive soft objects more diverse, this paper proposes ray-scaling operations to deform a primitive soft object. The proposed ray-scaling operations perform scaling via a given scaling object. But unlike scaling of affine transform which gives the same scaling factor to all the points, ray-scaling operations give each point in different directions an individual and a different scaling factor obtained from the ray-scaling object. As a result, by choosing suitable scaling objects, ray-scaling operations are able to erode or dilate a soft object locally with many special deformation effects. This paper also applies super-ellipsoids, super-quadrics and generalized distance functions to define a scaling object, and then successfully deforms a sphere into a six-balled, eight-balled or ten-balled object by using scaling objects of polyhedron, each of whose facets can deform to be a ball.
\end{abstract}

Key words: Field functions, blending operations, soft object modeling.

\section{Introduction}

In soft object modeling, a primitive soft object is defined as a level surface of field functions, called defined functions. Furthermore, primitive soft objects are connected smoothly by blending operations, such as Boolean set operators in [1]-[7] for creating a more complex objetct. Especially, because field function is required to decrease from 1 to 0 , soft objects can also be blended easily by performing addition only. Existing field functions can be found in [8]-[13].

To obtain primitive soft objects with more diverse shapes, in addition to developing new filed functions, deforming a soft object is a good method, such as affine transform [14] which are applied for translation, rotation, and scaling by using a matrix multiplication of a transformation matrix and a position vector instead of a point before calculating field function. However, unlike scaling of affine transform which performs a matrix multiplication on a point before calculating the function, this paper proposes an a new method for scaling called ray-scaling operations. A ray-scaling operation scales a soft object via a given scaling object by using a function operation of the defining functions of the soft object and the scaling object. Whereas scaling of affine transform only enlarge or shrink the deformed object proportionally and totally with the same scaling factor with respect to all the points in space, the proposed ray-scaling operation can scale a soft object with an individual and a different scaling factor assigned to each point in different directions, i.e. for any point, its scaling factor is decided by the intersection point of the scaling object and the vector from the origin to the point. Thus, by choosing suitable scaling objects, ray-scaling operations can deform (erode or dilate) a soft object locally, not entirely, with a lot of deformation effects. The remainder of this paper is organized as follows. Section 2 reviews some related works about soft objects. Section 3 introduces ray-scaling operations. Conclusions are given in Section 4. 


\section{Related Works}

\subsection{Soft Object Modeling}

A primitive soft object, is defined implicitly by using a field function $f_{i}(X): R^{3} \rightarrow R_{+}$as a defining function, and is represented by the point set

$$
\left\{X \in R^{3} \mid f_{i}(X)=0.5, i=1,2, \ldots,\right\},
$$

where $X \equiv(x, y, z) \in R^{3}$ and $R_{+} \equiv[0, \infty]$ in $R$. In the following, a soft object is denoted as $f_{i}(X)=0.5$ for short. And a field function $f_{i}(X)$ is usually defined as a composition of a potential function $P(d)$ and a distance function $d_{i}(X)$ by

$$
f_{i}(X)=\left(P \circ d_{i}\right)(X)=P\left(d_{i}(X)\right)
$$

where $P(d):[0,1] \rightarrow[1,0]$ decreases from 1 to 0 as $d$ increases from 0 to 1 and $P(0.5)=0.5$. Existing potential functions can be found in [10], [11], [13], [15]. Distance function $d_{i}(X)$ maps $R^{3}$ into [0, $\infty$ and is used to control the shape and size of soft object $f_{i}(X)=0.5$. In fact, $d_{i}(X)$ is represented using influential radius $R_{d}$ by

$$
d_{i}(X)=r / R_{d}=\overrightarrow{o X} / \overrightarrow{O I},
$$

where $R_{d}=\overrightarrow{o I}$ is called influential radius and $r=\overrightarrow{o X}$ is $\left(x^{2}+y^{2}+z^{2}\right)^{0.5}$, as in Fig. 1(a). $R_{d}$ is the distance from the origin to the intersecting point of $[x, y, \mathrm{z}]$ with the influential region $d_{i}(X)=1$. The subscript $d$ of $R_{d}$ is to symbolize that $R_{d}$ is an influential radius calculated by using $d(X)=1$ as the influential region. Thus, because $P(0.5)=0.5$ must hold, the shape of the soft object $f_{i}(X)=\left(P \circ d_{i}\right)(X)=0.5$ is the same as the shape $d_{i}(X)=0.5$.

Some of existing distance functions were presented such as:

- $L_{P}$ distance metrics [11], [12]:

$$
d(x, y, z)=\left(|x / a|^{p}+|y / b|^{p+}+z /\left.c\right|^{p}\right)^{1 / p}
$$

- Super-quadrics [9]:

$$
d(x, y, z)=\left(\left(|x / a|^{p 1}+|y / b|^{p 1}\right)^{\left.p 2 / p 1+|z / c|^{p 2}\right)^{1 / p 2}}\right.
$$

- Generalized distance functions [8]:

$$
d(x, y, z)=\left(\sum k_{i=1}\left|[x, y, z] \bullet \boldsymbol{n}_{i}\right|^{p}\right)^{1 / p}
$$

where $\bullet$ represents dot product operation and $\boldsymbol{n}_{i}, i=1, \ldots, k$, are unit normal vectors of planes.

\subsection{Blending Operations}

A Blending operation can create a more complex soft object by smoothly connecting $k$ primitive soft objects $f_{1}(X)=0.5, \ldots$, and $f_{k}(X)=0.5$ through a blending operator $B_{k}\left(x_{1}, \ldots, X_{k}\right): R_{+}{ }^{\prime} \rightarrow R_{+}$and it is written as the set

$$
\left\{X \in R^{3} \mid B_{k}\left(f_{1}(X), \ldots, f_{k}(X)\right)=0.5\right\} .
$$

Existing blending operators can be found in [1]-[7], which include Boolean set operations such as: Soft union blend [10], [11] by $B_{k}\left(x_{1}, \ldots, X_{k}\right)=x_{1}+x_{2}+\ldots+x_{k}$, super-ellipsoidal union by $B_{k}\left(x_{1}, \ldots, x_{k}\right)=\left(x_{1} p_{+} \ldots+x_{k}^{p}\right)^{1 / p}$ [7] and intersection blends [7] by $B_{k}\left(x_{1}, \ldots, X_{k}\right)=\left(x_{1}{ }^{p}+\ldots+X_{k}\right)^{1 / p}$ and $B_{k}\left(x_{1}, \ldots, X_{k}\right)=\left(x_{1}^{-p}+\ldots+x_{k}^{-p}\right)^{-1 / p}$. Fig. 1(b) shows a die created by Boolean set operations.

\subsection{Ray-Linear Functions}

This section presents a sufficient condition for a function to be a distance function.

Definition 1: A function $f(X): R^{3} \rightarrow R_{+}$is called non-negative ray-linear if $f(a X)=a f(X)$ holds for any $X \in R^{3}$ and $a \in R_{+}[15]$. For compactness, "ray-linear" stands for "non-negative ray-linear". Based on Definition 1, 
Theorem 1 was proposed in [4] to show how to be a distance function for a function or an operation:

- Theorem 1: If $f(X): R^{\mathrm{n}} \rightarrow R_{+}$is ray-linear, then $f(X)$ can be reformulated to be $r / R_{f}$, where $r=\|X\|$ and $R_{f}$ is influential radius. That is, $f(X)$ is qualified for being a distance function.

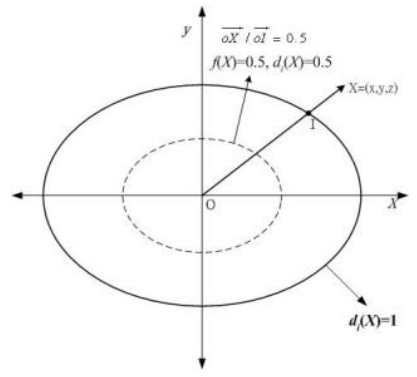

(a)

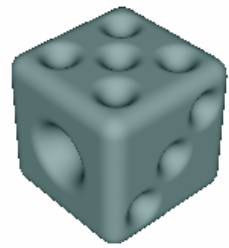

(b)

Fig. 1. (a). The influential region $d_{i}(X)=1$ and the shape $f_{i}(X)=0.5$, i.e. $d_{i}(X)=0.5$, dotted line. (b). A die created by using a difference operation of a cube from 21 spheres and the cube is an intersection of parallel planes.

\subsection{Overall Scaling of Affine Transform}

Let $M$ be a $4 \times 3$ transformation matrix:

$$
M_{4 \times 3}=\left[\begin{array}{ccc}
a & b & c \\
d & e & f \\
g & h & i \\
j & k & l
\end{array}\right]
$$

and $P$ be a $1 \times 4$ matrix $[x, y, z, 1]_{1 \times 4}$ where $[x, y, z]$ is a point $X$ in $R^{3}$. Then, an affine transformation $A(X): R^{3} \rightarrow R^{3}$ on a point $X$ is written by $A(X)=P M$. Thus, a soft object $f_{i}(X)=0.5$ can be deformed via affine transform [14] by

$$
f_{i}(A(X))=P\left(d_{i}(A(X))\right)=P\left(d_{i}(P M)\right)=0.5 .
$$

For example, when $a$, e and $I$ all equal $s$ in $M_{4 \times 3}$ and the other factors are $0, f_{i}(A(X))=0.5$ is viewed as overall scaling of $f_{i}(X)=0.5$, i.e. $f_{i}(A(X))=0.5$ is the same as $f_{i}(\boldsymbol{s} x, \boldsymbol{s} y, \boldsymbol{s} z)=f_{i}(\boldsymbol{s} X)=0.5$. However, deformation $f_{i}(\boldsymbol{s} X$, $s y, s z)=0.5$ can only enlarge or shrink a soft object $f_{i}(X)=0.5$ proportionally in all directions. For example, let $d(X)$ be a cube $\left(|x / 20|^{7}+|y / 20|^{7}+|z / 20|^{7}\right)^{1 / 7}$, then soft object $f_{i}(X)=\left(P \circ d_{i}\right)(X)=0.5$ is shown in Fig. $2(a)$ and it is enalrged by using $f_{i}(0.5 X)=0.5$ and shrinked by using $f_{i}(2 X)=0.5$ as shown in in Figs. 2 (b)-(c).

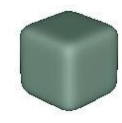

(a)

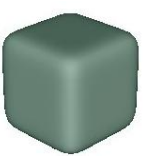

(b)

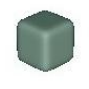

(c)

Fig. 2. Soft objects $f_{i}(X)=0.5, f_{i}(0.5 X)=0.5$ and $f_{i}(2 X)=0.5$ from left to right, where $d(X)=\left(|x / 20|^{7}\right.$

$$
\left.+|y / 20|^{7}+|z / 20|^{7}\right)^{1 / 7}
$$

\section{Ray-Scaling Operations}

This section proposes ray-scaling operations that can scale a soft object by offering every vector from the origin an individual scaling factor in all directions. As a result, the proposed ray-scaling operations can erode or dilate an soft object partially and locally.

Let $d(X)$ and $T(X)$ be ray-linear functions. Then, a ray-scaling operation of $d(X)$ by $T(X)$, denoted as 
$(d \otimes T)(X)$, is defined by:

$$
(d \otimes T)(X)=d(X)^{*}(T(X) / r)^{n},
$$

where $r=\left(x^{2}+y^{2}+z^{2}\right)^{0.5}$ and $n \in R$.

Furthermore, based on ray-scaling operation $(d \otimes T)(X)$, a soft object $(P \circ d)(X)=0.5$ ray-scaled by scaling object $T(X)=1$ is defined by

$$
f(X)=(P \circ(d \otimes T))(X)=0.5 .
$$

In (4), it is trivial to show $(d \otimes T)(X)$ is a ray-linear function. Since $(d \otimes T)(X)$ is ray-linear, it follows from Theorem 1 that $(d \otimes T)(X)$ can be reformulated and represented into the form $(d \otimes T)(X)=r / R_{d \otimes T}$. That is, by substituting $d(X)$ with $r / R_{d}$ and $T(X)$ with $r / R_{T}$ into (4), $(d \otimes T)(X)$ is rewritten by

$$
(d \otimes T)(X)=r / R_{d \otimes T}=r /\left(R_{d} / R_{T^{n}}\right) .
$$

Equation (5) indicates that for any $X$, the influential radius $R_{d \otimes T}$ of ray-scaling operation $(d \otimes T)(X)$ is $R_{d \otimes T}$ $=R_{d} / R_{T^{n}}$. This implies that when a ray-scaling operation $(d \otimes T)(X)$ is used as a distance function, then

- For any $X$, the influential radius $R_{d \otimes T}=R_{d} / R_{T}{ }^{n}$ for $X$ is different because the value $R_{T}$ for $X$, i.e. the influential radius of $T(X)$ for $X$, is different depending on the direction of vector $[x, y, z]$.

- Consequently, the surface $(d \otimes T)(X)=1$ is viewed as the surface $d(X)=1$ whose points $(x, y, z) \in d^{-1}(1)$ are scaled by different $R_{T^{n}}$, depending on $(x, y, z)$. That is, all the points $(x, y, z) \in d^{-1}(1)$ are scaled by an individual and different scaling factor $R_{T}{ }^{n}$.

Thus, by choosing a non-sphere ray-scaling object $T(X)=1$, a soft object $f(X)=(P \circ d)(X)=0.5$ can be deformed, not proportionally, by using ray-scaling operation $f(X)=(P \circ(d \otimes T))(X)=0.5$.

To obtain a non-sphere ray-scaling object $T(X)=1$, the following functions are qualified to be used as $T(X)$ because they all are ray-linear:

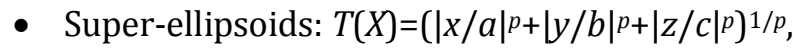

- Super-quadrics: $T(X)=\left(\left(|x / a|^{p 1}+|y / b|^{p 1}\right)^{p 2 / p 1}+|z / c|^{p 2}\right)^{1 / p 2}$,

- Generalized distance functions [2].

Depending on parameter $n$ in $(d \otimes T)(X)$ and the size and the shape of $T(X)=1,(d \otimes T)(X)$ are discussed as follows.

\subsection{When Parameter $n$ in $(d \otimes T)(X)$ Is Set Larger Than 1}

\subsubsection{When ray-scaling object $T(X)=1$ is bigger than a unit sphere}

When ray-scaling object $T(X)=1$ is bigger than a unit sphere, such as $T(X)=\left(|x|^{p}+|y|^{p}+|z|^{p}\right)^{1 / p}, p \geq 2$,

- Ray-scaling operation $(P \circ(d \otimes T))(X)=0.5$ shrinks or erodes soft object $(P \circ d)(X)=0.5$ locally.

- The eroding effect becomes more and more significant as $p$ increases from 2 to $\infty$.

- The eroding effect becomes more and more significant as $n$ in (5) increases from 1 to $\infty$.

- If scaling object $T(X)=1$ is a unit polyhedron, then $(P \circ(d \otimes T))(X)=0.5$ deforms and shrinks a sphere $(P$ 。 d) $(X)=0.5$ into a multi-balled object and every facet of the polyhedron generate a small ball.

All above are explained as follows. Let $T(X)$ be $\left(|x|^{p+}|y|^{p+|z|^{p}}\right)^{1 / p}$. When parameter $p$ is larger than $2, p>2$, scaling object $T(X)=1$ is bigger than a unit sphere. This means $R_{T}$ is always greater than 1 for any $X \in R^{3}$, and hence $R_{d} / R_{T^{n}}$ of $(d \otimes T)(X), R_{d \otimes T}$, becomes less than $R_{d}$ of $d(X)$. It follows that ray-scaling operation (P。 $(d \otimes T))(X)=0.5 \quad$ shrinks or erodes the soft object $(P \circ d)(X)=0.5$ locally when $p>2$ and $n \geq 1$.

Moreover, as parameter $p$ increases gradually from 2 to $\infty$, the scaling object $T(X)=\left(|x|^{p+}|y|^{p+|z| p}\right)^{1 / p=1}$ dilates and varies from a sphere to a cube, as shown in Fig. 3. This indicates that the eroding effect becomes more and more significant as $p$ increases from 2 to $\infty$. For example, let $d(X)$ be a sphere 


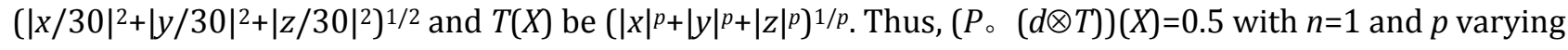
from $3,5,7$, to 10 erodes the sphere object $d(X)=0.5$ and make it change from a ball to a six-balled surface as in Fig. 4.

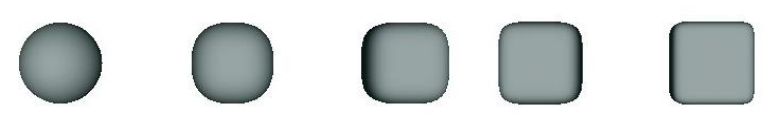

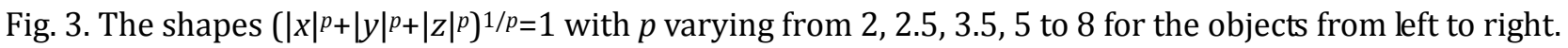

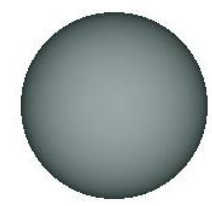

(a)
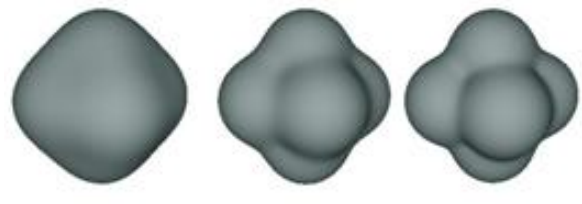

(b)

Fig. 4. (a). A sphere $(P \circ d)(X)=0.5$ where $d(X)=\left(|x / 30|^{2}+|y / 30|^{2}+|z / 30|^{2}\right)^{1 / 2}$. (b). Shape change of ray-scaling

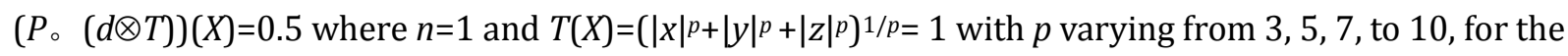
objects from left to right and the shapes $T(X)=1$ are seen in Fig. 4.

Besides, as parameter $n$ increases, the value of $R_{d \otimes T}=R_{d} / R_{T}{ }^{n}$ gets smaller. This is, as $n$ increases, the eroding effect becomes more and more significant, too. For example, following the case in Fig. 4 but with $p=7,(P \circ(d \otimes T))(X)=0.5$ erodes the object $(P \circ d))(X)=0.5$ into a six-balled object with deeper edges by varying $n$ from 1.5, 2, 2.5, to 3, as shown in Fig. 5.

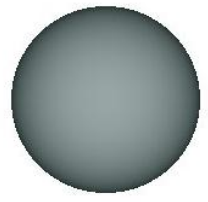

(a)

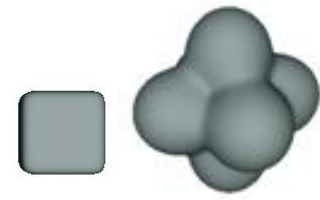

(b)

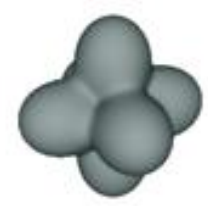

(c)
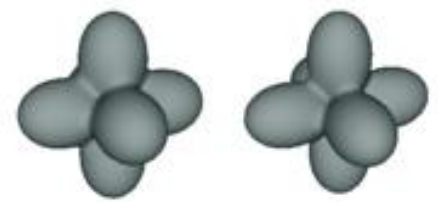

(c)

Fig. 5. (a). A sphere: $(P \circ d)(X)=0.5$ where $d(X)=\left(|x / 30|^{2}+|y / 30|^{2}+|z / 30|^{2}\right)^{1 / 2}$. (b). A scaling object $T(X)=\left(|x|^{p+}|y|^{p+}|z|^{p}\right)^{1 / p=1}$ with $p=7$. (c) The shape change of ray-scaling $(P \circ(d \otimes T))(X)=0.5$ of the sphere in

(a) by the cube in (b) with $n$ varying from 1.5, 2, 2.5, to 3 for the objects from left to right.

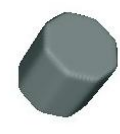

(a)

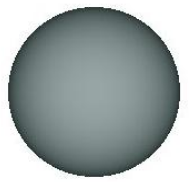

(b)

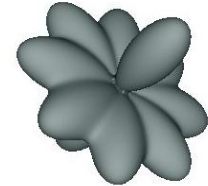

(c)

Fig. 6. (a). A polyhedron with ten facets defined by using a generalized distance function. (b). A sphere: $(P$ 。 d) $(X)=0.5$ where $d(X)=\left(|x / 30|^{2}+|y / 30|^{2}+|z / 30|^{2}\right)^{1 / 2}$. (c). A ray-scaling $(P \circ(d \otimes T))(X)=0.5$ of a sphere in (b) ray-scaled by the polyhedron $T(X)=1$ in (a).

More generally, if scaling object $T(X)=1$ is a unit polyhedron, then $(P \circ(d \otimes T))(X)=0.5$ deforms a sphere $(P \circ d)(X)=0.5$ into a multi-balled object and every facet of the polyhedron generate a ball. Fig. 6 shows a six-balled object because a cube with six-facets was used as the scaling object. Here is an example of 
ten-balled object. Let scaling object $T(X)=1$ be a polyhedron with ten facets, which is defined using a generalized distance function of normal vectors $\{[1,0,0],[0,1,0],[1 / \sqrt{2}, 1 / \sqrt{2}, 1],[-1 / \sqrt{2}, 1 / \sqrt{2}, 1],[0,0$, 1]\} and whose shape is shown in Fig. 6(a). Then, $(P \circ(d \otimes T))(X)=0.5$ with $n=7$ deforms the ball in Fig. 6(b) into a ten-balled object, as in Fig. 6(c).

\subsubsection{When ray-scaling object $T(X)=1$ is smaller than a unit sphere}

When ray-scaling object $T(X)=1$ is smaller than a unit sphere, such as $T(X)=\left(|x|^{p}+|y|^{p}+|z|^{p}\right)^{1 / p}, p<2$,

- Ray-scaling operation $(P \circ(d \otimes T))(X)=0.5$ enlarges or dilates soft object $(P \circ d)(X)=0.5$ locally.

- The dilating effect becomes more obvious as $p$ decreases from 2 to 1 or as $n$ in (5) increases from 1 to $\infty$

- If scaling object $T(X)=1$ is a rhombohedron, then $(P \circ(d \otimes T))(X)=0.5$ deforms and enlarge a sphere $(P$ 。 d) $(X)=0.5$ into a multi-balled object and every facet of the rhombohedron generate a big ball.

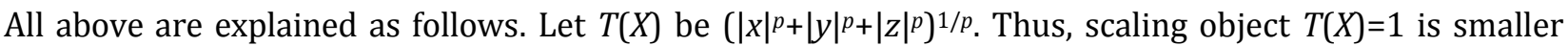
than a unit sphere when $p<2$ and $n \geq 1$, and as in Fig. 7, the shape $T(X)=\left(|x|^{p}+|y|^{p}+|z|^{p}\right)^{1 / p}=1$ shrinks from a sphere to be a rhombohedron. Because $R_{T}$ is always less than 1 for any $X \in R^{3}, R_{d} / R_{T}^{n}$ of $(d \otimes T)(X), R_{d \otimes T}$, is always greater than $R_{d}$ of $d(X)$. This implies that ray-scaling $(d \otimes T)(X)$ enlarge the object $d(X)=1$ when setting $p<2$ and $n \geq 1$. Especially, when the scaling object is a rhombohedron like the first object in Fig. 8, ray-scaling $(d \otimes T)(X)$ deforms a sphere into an eight-balled object because rhombohedron has eight facets.

For example, let $d(X)$ be a sphere $\left(|x / 20|^{2}+|y / 20|^{2}+|z / 20|^{2}\right)^{1 / 2}$ and $T(X)$ be $\left(|x|^{1.2}+|y|^{1.2}+|z|^{1.2}\right)^{1 / 1.2}$. As a result, $(d \otimes T)(X)=0.5$ enlarges the sphere $d(X)=0.5$ and become a 8-balled object, as in Fig. 8(c). In addition, as shown in Fig. 8(d), the dilation effect of $(d \otimes T)(X)$ on $d(X)=1$ become less and less obvious as parameter $p$ decreases from 2 to 1 . Fig. 8(d) shows ray-scaling $(P \circ(d \otimes T))(X)=0.5$ of a sphere $d(X)=\left(|x / 20|^{2}+|y / 20|^{2}+|z / 20|^{2}\right)^{1 / 2}$ by $T(X)=\left(|x|^{p}+|y|^{p+}+\left.z\right|^{p}\right)^{1 / p}$ with $n=1$ and $p$ varying from $1.2,1.4$, 1.6 , to 1.8 .

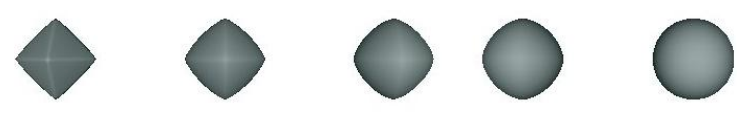

Fig. 7. The shapes of $\left(|x|^{p}+|y|^{p}+|z|^{p}\right)^{1 / p=1}$ and parameter $p$ varies from 1.1, 1.3, 1.5, 1.7 to 2 for the objects from left to right.

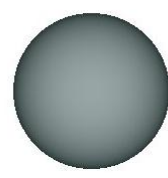

(a)

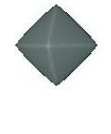

(b)

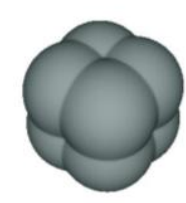

(c)
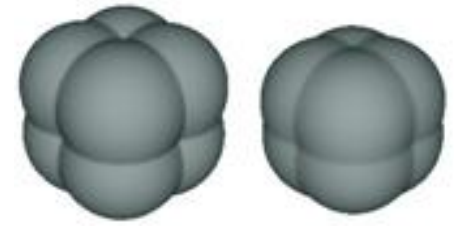

(d)
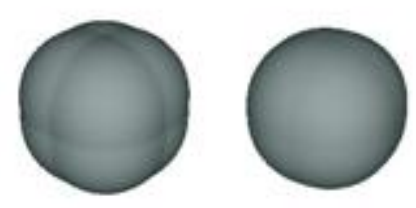

Fig. 8. (a). A sphere $d(X)=\left(|x / 20|^{2}+|y / 20|^{2}+|z / 20|^{2}\right)^{1 / 2}=1$. (b). A rhombohedron $T(X)=\left(|x|^{1.2+}+|y|^{1.2}+|z|^{1.2}\right)^{1 / 1.2}$ $=1$ with eight facets. (c). A ray-scaling $(P \circ(d \otimes T))(X)=0.5$ of the sphere in (a) by the object in (b). (d). The shape change of ray-scaling $(P \circ(d \otimes T))(X)=0.5$ of the sphere in (a) by $T(X)=\left(|x|^{p}+|y|^{p}+|z|^{p}\right)^{1 / p}$ with $n=1$ and $p$ varying from $1.2,1.4,1.6$, to 1.8 , where the dilation effect is becoming less obvious as $p$ decreases.

\subsection{When Parameter $n$ in $(d \otimes T)(X)$ is Set Less Than -1}

\subsubsection{When ray-scaling object $T(X)=1$ is bigger than a unit sphere}

When ray-scaling object $T(X)=1$ is bigger than a unit sphere,

- Ray-scaling operation $(P \circ(d \otimes T))(X)=0.5$ enlarges or dilates soft object $(P \circ d)(X)=0.5$ locally.

- The dilation effect becomes more obvious as $p$ increases from 2 to $\infty$ or as $n$ decreases from -1 to $-\infty$. 
All above are explained as follow. Let $T(X)$ be $\left(|x|^{p+}|y|^{p+}|z|^{p}\right)^{1 / p}$. Then, the scaling object $T(X)=1$ is bigger than a unit sphere when $p>2$. Thus, for $n<0, R_{d} / R_{T^{n}}$ of $(d \otimes T)(X), R_{d \otimes T}$, is always greater than $R_{d}$ of $d(X)$ for any $X \in R^{3}$. This implies that, when $p \geq 2$ and $n<0$, ray-scaling $(d \otimes T)(X)$ enlarges the shape $d(X)=1$. In addition, Fig. 10 shows how the dilation effect varies as $p$ increases gradually from 2 to $\infty$ or as $n$ decreases from -1 to

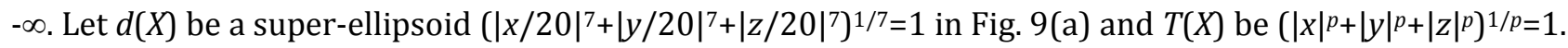
Fig. 9 (b) shows the dilation change of $(P \circ(d \otimes T))(X)=0.5$ with $n=-1$ and $p$ varying from 3,5 , to 7. Fig. $9(\mathrm{c})$ shows the dilation change of $(P \circ(d \otimes T))(X)=0.5$ with $p=4$ and $n$ varying from $-1.5,-1.8$, to -2 .

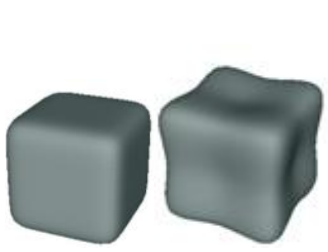

(a)

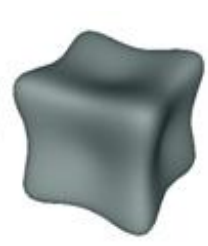

(b)

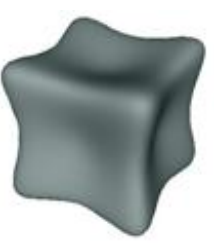

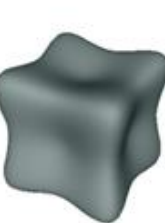

(1)

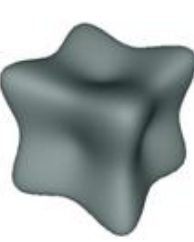

(c)

Fig. 9. (a). A cube, $\left(|x / 20|^{7}+|y / 20|^{7}+|z / 20|^{7}\right)^{1 / 7}=1$. (b). The shape change of ray-scaling $(P \circ(d \otimes T))(X)=0.5$ of the cube in (a) by $T(X)=\left(|x|^{p}+|y|^{p}+|z|^{p}\right)^{1 / p}$ with $n=-1$ and $p$ varying from 3,5 , to 7 . (c). The shape change of ray-scaling $(P \circ(d \otimes T))(X)=0.5$ of the cube in Fig. 10(a) by $T(X)=\left(|x|^{p}+|y|^{p+}+\left.z\right|^{p}\right)^{1 / p}$ with $p=4$ and $n$ varying from $-1.5,-1.8$, to -2 .

\subsubsection{When ray-scaling object $T(X)=1$ is smaller than a unit sphere}

When ray-scaling object $T(X)=1$ is smaller than a unit sphere,

- Ray-scaling operation $(P \circ(d \otimes T))(X)=0.5$ shrinks or erodes soft object $(P \circ d)(X)=0.5$ locally.

- The erosion effect becomes more obvious decreases from 2 to 1 or as $n$ in (5) decreases from -1 to $-\infty$.

Let $T(X)$ be $\left(|x|^{p}+|y|^{p}+|z|^{p}\right)^{1 / p}$. Scaling object $T(X)=1$ is smaller than a unit sphere when $p<2$. Thus, for $n<-1, R_{d} / R_{T}{ }^{n}$ of $(d \otimes T)(X), R_{d \otimes T}$, is always less than $R_{d}$ of $d(X)$ for any $X \in R^{3}$. This implies that ray-scaling $(P$ 。 $(d \otimes T))(X)=0.5$ shrinks soft object $(P \circ d)(X)=0.5$ when $p<2$ and $n<-1$.

\section{Conclusion}

In implicit surface modeling, affine transform enlarges or reduces an implicitly-defined soft object by performing a matrix multiplication. However, this kind of technique always give the same scaling factor to every point in 3D space, so it enlarges or reduces an object proportionally and totally in all directions. To conquer the problem, this paper has developed ray-scaling operations that can perform overall scaling via a scaling object defined by using a ray-linear function. The major differences of ray-scaling operations from affine transform are:

- Ray-scaling operations give an individual and different scaling factor, controlled by the chosen scaling object, to each point in different directions.

- Ray-scaling operations perform scaling by using a scaling object defined using a ray-linear function.

Thus, by choosing suitable scaling objects, ray-scaling operations are able to erode or dilate a soft object locally. In this paper, super-ellipsoids, super-quadrics and generalized distance functions have been applied successfully as scaling objects and they can deform, i.e. dilate or erode, a sphere into a multiple-balled (six-balled, eight-balled or ten-balled) object by using a polyhedron as a scaling object.

\section{Acknowledgment}

The author thanks Ministry of Science and Technology of Taiwan for the support of Project No. MOST 104-2221-E-366-008 and the work. 


\section{References}

[1] Bernhardt, A., Barthe, C., Cani, M.-0., \& Wyvill, B. (2010). Implicit blending revisited. Computer Graphics Forum, 29(2), 367-375.

[2] Groot, E., Wyvill, B., \& Wetering, H. (2009). Locally restricted blending of Blobtrees. Computers \& Graphics, 33(6), 690-697.

[3] Gourmel, O., Barthe, L., Cani, M., Wyvill, B., Bernhardt, A., Paulin, M., \& Grasberger, H. (2013). Gradient-based implicit blend. ACM Trans on Graphics, 32(2), 1-12.

[4] Hsu, P.-C., \& Lee, C. (2003). The scale method for blending operations in functionally based constructive geometry. Computer Graphics Forum, 22(2), 143-158.

[5] Hsu, P.-C. (2006). Implicit blends with an individual blending range control on every primitive's subsequent blend. Proceedings of International Conference Computer Graphics, Imaging and Visualization (pp. 534-541).

[6] Li, Q. (2007). Smooth piecewise polynomial blending operations for implicit shaped. Computer Graphics Forum, 26(2), 143-158.

[7] Ricii, A. (1973). A constructive geometry for computer graphics. The Computer Journal, 16(2), 157-160, May 1973.

[8] Akleman, E., \& Chen, J. (1999). Generalized distance functions. Proceedings of Shape Modeling International (pp. 72-79).

[9] Barr, A. H. (1981). Super-quadric. IEEE Computer Graphics and Applications, 1(1), 11-23.

[10] Blanc, C., \& Schlick, C. (1995). Extended field functions for soft objects. Proceedings of Implicit surfaces'95 (pp. 21-32).

[11] Blinn, J. F. (1982). A generalization of algebraic surface drawing. ACM Trans. on Graphics, 1(3), 235-256.

[12] Tiggers, M., Carpendale, M. S. T., \& Wyvill, B. (1999). Generalized distance metrics in implicit surface modeling. Proceedings of the Tenth Western Computer Graphics Symposium (pp. 14-18).

[13] Wyvill, G., McPheeters, C., \& Wyvill, B. (1986). Data structure for soft objects. The Visual Computer, 2(4), 227-234.

[14] Wyvill, B., Guy, A., \& Galin, E. (1998). Extending the CSG tree: Warping, blending and boolean operations in an implicit surface modeling systems. Proceedings of Implicit Surfaces'98 (pp. 128-136).

[15] Akleman, E. (1996). Interactive construction of smoothly blended star solids. Proceedings of the Graphics Interface (pp. 22-24).

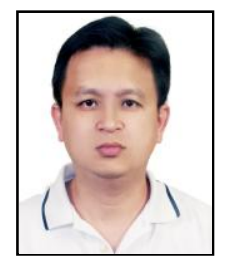

Pi-Chung Hsu was born in 1966. He received his doctor degree in information engineering at national Sun Yat-sen University, Taiwan in 2003. Now he is the associate professor at Shu-Te University, Taiwan. His research directions include computer graphics, and implicit surfaces modeling. 